\title{
Neurocysticercosis: understanding an infection
}

\section{Hector H Garcia}

Address: The Cysticercosis Working Group in Peru, School of Sciences, Universidad Peruana Cayetano Heredia; Department of Transmissible Diseases, Instituto de Ciencias Neurologicas, and Instituto Peruano de Parasitologia Clinica y Experimental (INPPACE), Lima, Peru

Email: Hector H Garcia - hgarcia@jhsph.edu

from Infectious diseases of the nervous system: pathogenesis and worldwide impact Paris, France. 10-13 September 2008

Published: 23 September 2008

BMC Proceedings 2008, 2(Suppl I):SI5

This abstract is available from: http://www.biomedcentral.com/I753-656I/2/SI/SI5

(c) 2008 Garcia; licensee BioMed Central Ltd.

Neurocysticercosis is the infection of the human central nervous system by the larvae (cysticerci) of the pork tapeworm Taenia solium. Cysticercosis is endemic in most developing countries and cases are seen in industrialized countries (over 1000 per year in the US only) due to immigration from endemic regions. Cysticercosis has been classically conceptualized as a neurological disease and labeled as a single entity. This approach led to much confusion and many difficulties in understanding disease evolution, prognosis, management, and performance of specific serological and imaging tests. Data will be presented showing the particular characteristics of each subtype of neurocysticercosis and the different spectrum of serological responses found in villagers in endemic communities (mostly neurologically asymptomatic) compared to clinical cases as seen in health centers. Understanding that cysticercosis is an infection and symptomatic neurocysticercosis represents the tip of the clinical iceberg, together with a clear definition of specific subtypes of disease based on the location and stage of the parasites allows a better understanding of its epidemiology and pathophysiology, and would also contribute to sound use of antiparasitic medication. 